\title{
Optimal Investment for Insurers with the Extended CIR Interest Rate Model
}

\author{
Mei Choi Chiu' ${ }^{1}$ and Hoi Ying Wong ${ }^{2}$ \\ ${ }^{1}$ Department of Mathematics and Information Technology, Hong Kong Institute of Education, Hong Kong \\ ${ }^{2}$ Department of Statistics, The Chinese University of Hong Kong, Hong Kong
}

Correspondence should be addressed to Mei Choi Chiu; mcchiu@ied.edu.hk

Received 25 January 2014; Accepted 14 May 2014; Published 30 June 2014

Academic Editor: Guanglu Zhou

Copyright ( 2014 M. C. Chiu and H. Y. Wong. This is an open access article distributed under the Creative Commons Attribution License, which permits unrestricted use, distribution, and reproduction in any medium, provided the original work is properly cited.

\begin{abstract}
A fundamental challenge for insurance companies (insurers) is to strike the best balance between optimal investment and risk management of paying insurance liabilities, especially in a low interest rate environment. The stochastic interest rate becomes a critical factor in this asset-liability management (ALM) problem. This paper derives the closed-form solution to the optimal investment problem for an insurer subject to the insurance liability of compound Poisson process and the stochastic interest rate following the extended CIR model. Therefore, the insurer's wealth follows a jump-diffusion model with stochastic interest rate when she invests in stocks and bonds. Our problem involves maximizing the expected constant relative risk averse (CRRA) utility function subject to stochastic interest rate and Poisson shocks. After solving the stochastic optimal control problem with the HJB framework, we offer a verification theorem by proving the uniform integrability of a tight upper bound for the objective function.
\end{abstract}

\section{Introduction}

The random movement of interest rates generates challenges to the asset-liability management practice for insurance companies (insurers). For instance, Thind [1] reports the potential problems recently faced by Nordic insurers associated with the interest rate movement. Actually, similar problems occur in other markets as well and the insurance industry has increasing demand in quantitative methods for managing interest rate risk in their investment portfolios.

Two major distinguished features of insurers' portfolios are the long-term investment horizon and the risk of paying out insurance claims. As life insurance contracts and pension plans are often long-term commitments, insurers have to plan their investment with a long-term horizon in mind. As such, the stochastic interest rate model adopted should stay positive throughout the long-term investment horizon and possibly gets close to zero, which is exactly the current economic situation. The interest model proposed by Cox et al. [2] (CIR) is constructed for this purpose. An even more realistic consideration allows parameters in the CIR model to be time-varying and this constitutes the extended CIR model. The positivity of CIR model also makes it a model for describing stochastic volatility. Wong and Chiu [3] offer a review and recent advances on the use of CIR model in stochastic volatility. However, Deelstra et al. $[4,5]$ are the pioneers who investigated the optimal investment problems with and without a minimum guarantee under the extended CIR model. Ferland and Watier [6] further analyzed the mean-variance efficiency of utility maximization problem under the extended CIR model. It is recently extended to incorporate stochastic volatility in [7].

While the literature has already investigated long-term optimal investment problems with the extended CIR model, the incorporation of insurance claim payment is yet to be considered in the present paper. The insurance claims are used to be modeled by a compound Poisson process in actuarial science. Taking insurance claims into account makes the insurer's wealth become a jump-diffusion model with an extended CIR stochastic interest rate. This kind of stochastic model is not considered in the utility maximization problem nor in the insurance literature, to the best of our knowledge. It is shown in [8-10] that optimal investment associated with jump-diffusion models could be challenging mathematical 
problems. The problem considered in the present paper even adds the stochastic interest rate.

This paper contributes to the literature by deriving the closed-form solution to the optimal insurer's investment problem with the extended CIR interest rate model and offering a verification theorem to the corresponding $\mathrm{HJB}$ equation. The mathematical difficulty arises from the jumpdiffusion model and the stochastic interest rate in the insurer's wealth process that makes the HJB equation a nonlinear partial integral differential equation (PIDE). While solving the PIDE is the first challenge, proving the verification theorem for the solution of the PIDE being the eligible optimal value function is another. The verification theorem is deduced using the recent framework of Chiu and Wong [10] when there is a mean-reverting stochastic variable, which is the interest rate in our case.

The remaining part of the paper is organized as follows. Section 2 presents the problem formulation and defines the problem of interest. Section 3 solves the optimal control problem and proves the verification theorem. Concluding remarks are made in Section 4.

\section{Problem Formulation}

2.1. The Financial Market. Consider a financial market in which $n+2$ assets are traded continuously within the time horizon $[0, T]$. These assets are labeled by $S_{i}$, stock $i$, for $i=$ $0,1,2, \ldots, n$ and a zero-coupon bond $B$. Here, $S_{0}$ denotes a risk-free asset.

The risk-free asset satisfies the differential equation:

$$
\begin{gathered}
d S_{0}(t)=r(t) S_{0}(t) d t, \\
S_{0}(0)=s_{0}>0,
\end{gathered}
$$

where the stochastic short rate follows the CIR model [2]:

$$
\begin{gathered}
d r(t)=(a(t)-b(t) r(t)) d t-\sigma_{r}(t) \sqrt{r(t)} d W_{t}^{r}, \\
r(0)=r_{0}>0,
\end{gathered}
$$

in which $W_{r}(t)$ is a Wiener process and $a(t), b(t)$, and $\sigma_{r}(t)$ are time-deterministic functions.

The risky assets, $S_{1}(t), \ldots, S_{n}(t)$, satisfy the stochastic differential equation (SDE):

$$
\begin{array}{r}
d S_{i}(t) \\
=S_{i}(t)\left\{r(t) d t+\left[\sum_{j=1}^{n}\left(\Sigma_{s}(t)\right)_{i j}\left((\Lambda(t))_{j} d t+d W_{t}^{S_{j}}\right)\right]\right. \\
\left.+\left(\Sigma_{r}(t)\right)_{i} \sqrt{r(t)}\left(\lambda_{r}(t) \sqrt{r(t)} d t+d W_{t}^{r}\right)\right\}, \\
t \in[0, T] ;
\end{array}
$$$$
S_{i}(0)=S_{0}^{i} \text {, }
$$

where $W_{t}^{s}=\left(W_{t}^{S_{1}}, \ldots, W_{t}^{S_{n}}\right)^{\prime}$ is a standard $\mathscr{F}_{t \geq 0}$-adapted $n$-dimensional Wiener process on a fixed filtered complete probability space $\left(\Omega, \mathscr{F}, \mathscr{P}, \mathscr{F}_{t \geq 0}\right)$ with $n>m, W_{t}^{S_{i}}$ and $W_{t}^{S_{j}}$ are mutually independent for all $i \neq j$, and $W_{t}^{S_{i}}$ and $W_{t}^{r}$ are mutually independent for all $i \in\{1, \ldots, n\}$. Denote $W_{t}=$ $\left(W_{t}^{S_{1}}, \ldots, W_{t}^{S_{n}}, W_{t}^{r}\right)^{\prime} . \mathbb{F}:=\left\{\mathscr{F}_{t}\right\}_{t \geq 0}$ is the filtration generated by $W_{t}$ augmented by the null sets of $\mathscr{P}, \Sigma_{s}(t)$ is the volatility matrix of stocks defined in the Banach space of $\mathbb{R}^{n \times n}$-valued continuous function on $[0, T]$ such that the nondegeneracy condition of $\Sigma_{s}(t) \Sigma_{s}(t) \geq \delta I_{m}$ holds for all $t \in[0, T]$ and for some $\delta>0$. The term $\Sigma_{r}(t) \sqrt{r(t)}$ is the volatility factor of stock prices contributed by the interest rate while $(\Lambda(t))_{j}$ and $\lambda_{r}(t) \sqrt{r(t)}$ are the market price of risk of the risky asset $j$ and the market price of interest rate risk, respectively. This financial market setting is also considered in [4-6].

Under the CIR model, the zero-coupon bond price at time $t$ with maturity $T$ evolves as

$d B(t, T)$

$$
\begin{gathered}
=B(t, T)\left[r d t+\sigma_{B}(t)\left(\lambda_{r}(t) \sqrt{r(t)} d t+d W_{t}^{r}\right)\right], \\
B(T, T)=1,
\end{gathered}
$$

where $\sigma_{B}(t)=\sigma_{r}(t) h(t, T) \sqrt{r(t)}$ and $h(t, T)$ solves the ordinary differential equation (ODE):

$$
\begin{aligned}
\frac{d h(t, T)}{d t}= & \frac{1}{2} \sigma_{r}(t)^{2} h(t, T)^{2}+\left(b(t)-\lambda_{r}(t) \sigma_{r}(t)\right) \\
& \times h(t, T)-1, \quad h(T, T)=0 .
\end{aligned}
$$

The interest model in (2) is known as the extended CIR model; see [6]. The technical conditions below ensure a positive interest rate process from (2).

Assumption 1. $\left(\mathrm{H}_{0}\right) a(t), b(t), \sigma_{r}(t), \lambda_{r}(t)$, and $\Lambda(t)$ are locally bounded for $t \in[0, T]$.

$\left(\mathrm{H}_{1}\right) 2 a(t) \geq \sigma_{r}^{2}(t)$ for any $t \in[0, T]$.

$\left(\mathrm{H}_{2}\right) a(t), b(t) \geq 0, \inf _{0 \leq t \leq T} \sigma_{r}(t)>0$ for any $T>0$, and $\sigma_{r}(t)$ is continuously differentiable.

2.2. Insurer's Wealth. The classical risk process assumes that an insurer's wealth evolves as

$$
Y(t)=Y_{0}+C(t)-L(t),
$$

where $Y_{0}$ is the initial wealth, the nonnegative deterministic function $C(t)$ is the accumulated insurance premium, and $L(t)$ represents accumulated random payments for insurance claims.

Assumption 2. The accumulated random payments (insurance liabilities) follow a compound Poisson process,

$$
L(t)=\int_{0}^{t} Y(s)\left(1-e^{-z}\right) d \mathcal{N}_{s}, \quad L(0)=0,
$$

where $\left\{\mathcal{N}_{t}, 0 \leq t \leq T\right\}$ is the Poisson process with intensity $\mu(t)$, where the moment generating function of $\int_{\tau_{1}}^{\tau_{2}} \mu(t) d t$ 
exists for $0 \leq \tau_{1} \leq \tau_{2} \leq T,\left(1-e^{-z}\right)$ is an insurance claim portion at time $t$, and $z$ is a nonnegative random variable independent of $\mathcal{N}_{t}$ and $W_{t}$ and has a well-defined moment generating function.

The risk model (6) ignores the fact that insurance companies usually participate in the financial market by investing in stocks and bonds; see comments in [11]. Hence, our model allows insurers to do so. Assume for the moment that the insurance premium $C(t) \equiv 0$ to simplify the mathematical setup, but the situation in which the premium is positive can be fully addressed using arguments similar to $[9,10]$.

Let $u_{i}(t)$ and $u_{B}(t)$ be the cash amount invested in stock $i$ and the zero-coupon bond, respectively, and let $N_{i}(t)$ and $N_{B}(t)$ be the numbers of holding units in stock $i$ and the zerocoupon bond in the portfolio of the insurer, respectively. The insurer's wealth level at time $t$ is

$$
\begin{aligned}
Y(t) & =\sum_{i=0}^{n} u_{i}(t)+u_{B}(t) \\
& =\sum_{i=0}^{n} N_{i}(t) S_{i}(t)+N_{B}(t) B(t, T), \quad Y(0)=Y_{0} .
\end{aligned}
$$

The ALM strategy $u(t)=\left(u_{1}(t), \ldots, u_{n}(t), u_{B}(t)\right)^{\prime} \quad:=$ $\left(u_{s}(t), u_{B}(t)\right)^{\prime}$ is said to be admissible if $u(t)$ is a nonanticipating process such that $\mathrm{E}\left[\int_{0}^{T}\|u(\tau)\|^{2} d \tau\right]<\infty$.

Definition 3. Define $\Pi$ as the space collecting all admissible trading strategies.

Unlike traditional portfolio selection problems, we do not require $u(t)$ to be self-financing because there are interim random insurance payments $d L(t)$. Specifically, the budget equation of the insurer's wealth is $[8,11]$

$$
\begin{aligned}
d Y(t)= & \sum_{i=0}^{n} N_{i}(t) d S_{i}(t)+N_{B}(t) d B(t, T) \\
& +\sum_{i=0}^{n} S_{i}(t) d N_{i}(t)+B(t, T) d N_{B}(t) \\
= & \sum_{i=0}^{n} N_{i}(t) d S_{i}(t)+N_{B}(t) d B(t, T)-d L(t) .
\end{aligned}
$$

Thus, the insurer draws an amount of $\sum_{i=0}^{n} S_{i}(t) d N_{i}(t)+$ $B(t, T) d N_{B}(t)$ from the portfolio to finance an insurance claim $d L$. Assumption 2 implies that

$$
\begin{aligned}
d Y(t)= & \sum_{i=0}^{n} u_{i}(t) \frac{d S_{i}(t)}{S_{i}(t)}+u_{B}(t) \frac{d B(t, T)}{B(t, T)} \\
& -Y\left(1-e^{-z}\right) d \mathcal{N}_{t} .
\end{aligned}
$$

Applying Itô's lemma, SDEs (2), (3), and (4), the wealth process is given by

$$
\begin{aligned}
d Y(t)=[ & r(t) Y(t)+u_{s}(t)^{\prime} \\
& \times\left(\Sigma_{s}(t) \Lambda(t)+\Sigma_{r}(t) \lambda_{r}(t) r(t)\right) \\
& \left.+u_{B}(t) \sigma_{B}(t) \lambda_{r}(t) \sqrt{r(t)}\right] d t \\
& +u_{s}(t)^{\prime} \Sigma_{s}(t)(t) d W_{t}^{s} \\
& +\left(u_{s}(t)^{\prime} \Sigma_{r}(t) \sqrt{r(t)}+u_{B}(t) \sigma_{B}(t)\right) d W_{t}^{r} \\
& -Y\left(1-e^{-z}\right) d \mathcal{N}_{t}, \quad Y(0)=Y_{0},
\end{aligned}
$$

in which $\mathcal{N}_{t}$ is a doubly stochastic Poisson process with $\mathbb{F}$-predictable nonnegative intensity $\mu(t)$; the parameters $\left(\Sigma_{s}(t)\right)_{i j},\left(\Sigma_{r}(t)\right)_{i}, z$, and $\mu(t)$ are uniformly bounded and $\mathbb{F}$ predictable on $[0, T]$, for $i=1, \ldots, n$ and $j=1, \ldots, n$. Define $\mathbb{H}:=\left\{\mathscr{H}_{t}\right\}_{t \geq 0}$ the filtration generated by $\mathcal{N}(t)$ augmented by

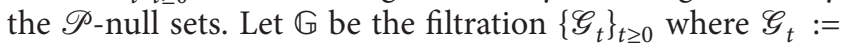
$\mathscr{F}_{t} \vee \mathscr{H}_{t}$, the smallest filtration containing $\mathbb{F}$ and $\mathbb{H}$. Note that $\mathscr{G}_{t}$ can be regarded as the information available to the investor at time $t$. Define the compensated Poisson process $\mathscr{M}_{t}:=\mathscr{N}_{t}-\int_{0}^{t} \mu(s) d s$, which is a $\mathbb{G}$-martingale.

2.3. Optimal Investment for Insurer. In economics, one school of thought on optimal investment decisions suggests maximizing the expected utility of an investor's future wealth, $\mathrm{E}\left[U\left(Y_{T}\right)\right]$. The standard approach assumes the utility to be strictly increasing and concave. If the utility function is twice differentiable, then $U^{\prime}(y)>0$ and $U^{\prime \prime}(y)<0$ for all $y$. A popular choice of utility is the CRRA utility function which takes the following form:

$$
\begin{gathered}
U(y)=\frac{y^{1-\gamma}-1}{1-\gamma} \quad \text { for } \gamma \in(0,1), \\
U(y)=\ln y \quad \text { for } \gamma=1 .
\end{gathered}
$$

Therefore, we consider the following research problem:

$$
\begin{array}{lll}
\text { Research Problem } & \max _{u(\cdot)} \mathrm{E}[U(Y(T))] \\
& \text { s.t. } & (2),(11), u(\cdot) \in \Pi,
\end{array}
$$

where $U(y)$ is defined in (12).

If $r(t)$ is a time-deterministic function and the Poisson process is absent in (11), then the corresponding utility portfolio problem is reduced to the standard utility portfolio optimization problem or the Merton problem. Unfortunately, $r(t)$ is stochastic and the insurance liability follows a compound Poisson process within our consideration. Therefore, the wealth process (11) resembles a jump-diffusion model with a random drift.

\section{The Optimal Solution}

We divide our derivation into two cases. In the first case, $\gamma<1$ so that the utility function is proportional to a power 
function. It refers to a power utility. In the second case, we take the limit of $\gamma \rightarrow 1$ on the CRRA utility. As the limit is the logarithmic function, it refers to the logarithmic utility or Bernoulli's utility function.

3.1. Power Utility. In our research problem, the optimal decision is not affected by adding a real constant to the objective function. The power utility maximization problem can then be reduced to

$$
\max \mathrm{E}\left[\frac{Y(T)^{1-\gamma}}{1-\gamma}\right]
$$

where the insurer's wealth follows the SDE in (11).

Theorem 4. Under Assumptions 1 and 2, the research problem (13) with the power utility (14) has the optimal solution (investment policy),

$$
\begin{gathered}
u_{s}^{*}(t, Y(t), r(t))=\frac{1}{\gamma}\left(\Sigma_{s}^{\prime}(t)\right)^{-1} \Lambda(t) Y(t) \\
u_{B}^{*}(t, Y(t), r(t))=\frac{\sqrt{r(t)}}{\gamma \sigma_{B}(t)}\left(\lambda_{r}(t)-\Lambda^{\prime}(t) \Sigma_{s}(t)^{-1} \Sigma_{r}(t)\right. \\
\left.-K(t, T) \sigma_{r}(t)\right) Y(t)
\end{gathered}
$$

and the optimal value of the objective function

$$
\begin{aligned}
& \left.\mathrm{E}\left[\frac{Y(T)^{1-\gamma}}{1-\gamma} \mid \mathscr{G}_{0}\right]\right|_{u^{*} u^{*}} \\
& \quad=\frac{Y_{0}^{1-\gamma}}{1-\gamma} \exp \left[K(0, T) r(0)+M^{\prime}(0, T)\right],
\end{aligned}
$$

where $K(t, T)$ and $M(t, T)$ satisfy the system of ordinary differential equations (ODE):

$$
\begin{gathered}
\dot{K}(t, T)-\left(b(t)+\frac{1-\gamma}{\gamma} \sigma_{r}(t) \lambda_{r}(t)\right) K(t, T) \\
+\frac{\sigma_{r}(t)^{2}}{2 \gamma} K(t, T)^{2}+\left(1-\gamma+\frac{1-\gamma}{2 \gamma} \lambda_{r}(t)^{2}\right)=0, \\
K(T, T)=0 \\
\dot{M}(t, T)+a(t) K(t, T)+\mathrm{E}\left[\left(e^{-z(1-\gamma)}-1\right) \mu(t)\right] \\
-\frac{1-\gamma}{2 \gamma}\|\Lambda(t)\|^{2}=0 \\
M(T, T)=0 .
\end{gathered}
$$

Proof. The proof is based on the classic HJB framework. Let

$$
V(t, y, r)=\sup _{u \in \Pi} \mathrm{E}\left[U\left(Y^{u}(T)\right) \mid \mathscr{G}_{t}\right] .
$$

For a fixed terminal time $T$, the corresponding HJB equation is

$$
\begin{aligned}
& V_{t}+V_{r}^{\prime}(a-b r)+\frac{1}{2} \operatorname{tr}\left(V_{r r} \sigma_{r}^{2} r\right) \\
&+\mathrm{E}\left[\left(V\left(t, y-y\left(1-e^{-z}\right), r\right)-V(t, y, r)\right) \mu\right] \\
&+\sup _{u}\left\{V_{y}\left(r y+u_{s}^{\prime}\left(\Sigma_{s} \Lambda+\Sigma_{r} \lambda_{r} r\right)+u_{B} \sigma_{B} \lambda_{r} \sqrt{r}\right)\right. \\
& \quad-V_{r y} \sigma_{r} \sqrt{r}\left(u_{s}^{\prime} \Sigma_{r} \sqrt{r}+u_{B} \sigma_{B}\right) \\
&+\frac{1}{2} V_{y y}\left(u_{s}^{\prime} \Sigma_{s} \Sigma_{s}^{\prime} u_{s}+u_{s}^{\prime} \Sigma_{r} \Sigma_{r}^{\prime} u_{s} r\right. \\
&\left.\left.+u_{B}^{2} \sigma_{B}^{2}+2 u_{s}^{\prime} \Sigma_{r} \sqrt{r} u_{B} \sigma_{B}\right)\right\}=0
\end{aligned}
$$

with $V(T, y, r)=y^{1-\gamma} /(1-\gamma)$. Thus, the optimal feedback control, $u^{*}$, maximizes

$$
\begin{array}{r}
V_{y} u^{\prime}\left(\begin{array}{c}
\Sigma_{s} \Lambda+\Sigma_{r} \lambda_{r} r \\
\sigma_{B} \lambda_{r} \sqrt{r}
\end{array}\right)-V_{r y} \sigma_{r} \sqrt{r} u^{\prime}\left(\begin{array}{c}
\Sigma_{r} \sqrt{r} \\
\sigma_{B}
\end{array}\right) \\
+\frac{1}{2} V_{y y} u^{\prime}\left(\begin{array}{cc}
\Sigma_{s} \Sigma_{s}^{\prime}+\Sigma_{s} \Sigma_{s}^{\prime} r & \Sigma_{r} \sigma_{B} \sqrt{r} \\
\Sigma_{r}^{\prime} \sigma_{B} \sqrt{r} & \sigma_{B}^{2}
\end{array}\right) u,
\end{array}
$$

where $u=\left(u_{s} u_{B}\right)^{\prime}$. If $V_{y y}<0$, differentiating (21) with respect to $u$ and setting the differential to zero results in

$$
u^{*}=\Sigma^{-1}\left(\frac{V_{r y}}{V_{y y}} \sigma_{r} \sqrt{r}\left(\begin{array}{c}
\Sigma_{r} \sqrt{r} \\
\sigma_{B}
\end{array}\right)-\frac{V_{y}}{V_{y y}}\left(\begin{array}{c}
\Sigma_{s} \Lambda+\Sigma_{r} \lambda_{r} r \\
\sigma_{B} \lambda_{r} \sqrt{r}
\end{array}\right)\right),
$$

where $\Sigma=\left(\begin{array}{cc}\Sigma_{s} \Sigma_{s}^{\prime}+\Sigma_{s} \Sigma_{s}^{\prime} r & \Sigma_{r} \sigma_{B} \sqrt{r} \\ \Sigma_{r}^{\prime} \sigma_{B} \sqrt{r} & \sigma_{B}^{2}\end{array}\right)$. Otherwise, if $V_{y y} \geq 0$, then the optimization has no solution.

Note that $\Sigma^{-1}$ can be simplified by matrix inversion lemma (or called Sherman-Morrison-Woodbury formula). Hence, we have

$$
\Sigma^{-1}=\left(\begin{array}{cc}
\left(\Sigma_{s} \Sigma_{s}^{\prime}\right)^{-1} & -\frac{\sqrt{r}}{\sigma_{B}}\left(\Sigma_{s} \Sigma_{s}^{\prime}\right)^{-1} \Sigma_{r} \\
-\frac{\sqrt{r}}{\sigma_{B}} \Sigma_{r}^{\prime}\left(\Sigma_{s} \Sigma_{s}^{\prime}\right)^{-1} & \frac{1+r \Sigma_{r}^{\prime}\left(\Sigma_{s} \Sigma_{s}^{\prime}\right)^{-1} \Sigma_{r}}{\sigma_{B}^{2}}
\end{array}\right) .
$$

Therefore, (22) becomes

$$
u^{*}=\left(\begin{array}{c}
u_{s}^{*} \\
u_{B}^{*}
\end{array}\right)=\left(\begin{array}{c}
-\frac{V_{y}}{V_{y y}}\left(\Sigma_{s}^{\prime}\right)^{-1} \Lambda \\
\frac{\sqrt{r}}{\sigma_{B}}\left[\frac{V_{r y}}{V_{y y}} \sigma_{r}-\frac{V_{y}}{V_{y y}}\left(\lambda_{r}-\Lambda^{\prime} \Sigma_{s}^{-1} \Sigma_{r}\right)\right]
\end{array}\right) .
$$


Substituting the $u^{*}$ into the HJB equation (20), the PIDE of $V$ becomes

$$
\begin{aligned}
V_{t}+ & V_{r}(a-b r)+\frac{1}{2} V_{r r} \sigma_{r}^{2} r+V_{y} r y \\
& +\mathrm{E}\left[\left(V\left(t, y-y\left(1-e^{-z}\right), r\right)-V\right) \mu\right] \\
& -\frac{1}{2} V_{y y}\left[\frac{V_{r y}}{V_{y y}} \sigma_{r} \sqrt{r}\left(\begin{array}{c}
\Sigma_{r} \sqrt{r} \\
\sigma_{B}
\end{array}\right)-\frac{V_{y}}{V_{y y}}\left(\begin{array}{c}
\Sigma_{s} \Lambda+\Sigma_{r} \lambda_{r} r \\
\sigma_{B} \lambda_{r} \sqrt{r}
\end{array}\right)\right]^{\prime} \\
& \times \Sigma^{-1}\left[\frac{V_{r y}}{V_{y y}} \sigma_{r} \sqrt{r}\left(\begin{array}{c}
\Sigma_{r} \sqrt{r} \\
\sigma_{B}
\end{array}\right)-\frac{V_{y}}{V_{y y}}\left(\begin{array}{c}
\Sigma_{s} \Lambda+\Sigma_{r} \lambda_{r} r \\
\sigma_{B} \lambda_{r} \sqrt{r}
\end{array}\right)\right]
\end{aligned}
$$$$
=0 \text {, }
$$

with terminal condition $V(T, y, r)=U(y)$. As $U(y)=$ $y^{1-\gamma} /(1-\gamma)$, consider an exponential affine form for $V$ :

$$
V(t, y, r)=\frac{y^{1-\gamma}}{1-\gamma} \exp [K(t, T) r+M(t, T)]
$$

where $K$ and $M$ are deterministic functions of $t$ and satisfy the ODEs (17) and (18), respectively. Note that the ODEs (17) can be regarded as a Riccati equation. Clearly, the terminal value of the function in (26) satisfies the terminal condition in $(25)$ and $V_{y y}<0$. Taking partial derivatives to the affine form $V$ with respect to $t, y$, and $r$, we have

$$
\begin{array}{cc}
V_{t}=(\dot{K} r+\dot{M}) V ; & V_{r}=K V ; \\
V_{y}=\frac{1-\gamma}{y} V ; & V_{r r}=K^{2} V ;
\end{array}
$$

$$
\begin{gathered}
V_{y y}=-\frac{\gamma(1-\gamma)}{y^{2}} V ; \quad V_{r y}=\frac{1-\gamma}{y} K V ; \\
\mathrm{E}\left[\left(V\left(t, y-y\left(1-e^{-z}\right), r\right)-V\right) \mu\right] \\
=\mathrm{E}\left[\left(e^{-z(1-\gamma)}-1\right) \mu\right] V .
\end{gathered}
$$

After substituting these expressions into the left-hand side of (25), simple but tedious calculations easily verify that the proposed solution form satisfies the PIDE in (25). Thus, the solution form in (26) is actually a solution of the PDE in (25). As the value function is twice continuously differentiable and all of the parameters are uniformly bounded and predictable, the classical verification theorems of [12] (III, Theorem 8.1) confirm that the proposed affine form of value function in (26) and the control in (15) are the optimal value function and optimal feedback control, respectively.

Theorem 4 asserts that if we are able to solve the system of ODEs (17) and (18), then both optimal investment policy $u^{*}$ and the optimal function value are efficiently computed. In fact, the solution to (18) is simple given the solution of (17) because (18) is linear ODE. However, ODE (17) is a Riccati differential equation (RDE). Using Radon's lemma (c.f. Theorem 3.1.1 of the book [13]), the matrix RDE (17) can be solved systematically.

Proposition 5. If $K(t, T)$ satisfies the matrix $R D E$ (17) and $M(t, T)$ satisfies the linear ODE in (18), then the solution is explicitly obtained as follows.

(1) $K(\tau):=K(t, T)=R_{2}(\tau) R_{1}^{-1}(\tau)$, where $\tau=T-t$, and $R=\left(R_{1}(\tau)^{\prime} R_{2}(\tau)^{\prime}\right)^{\prime}$ is the solution of the linear system of ODEs in the interval $[0, T]$ :

$$
\begin{aligned}
& \frac{d R}{d \tau}=\left(\begin{array}{cc}
\frac{1}{2}\left(b(t)+\frac{1-\gamma}{\gamma} \sigma_{r}(t) \lambda_{r}(t)\right) & -\frac{\sigma_{r}(t)^{2}}{2 \gamma} \\
1-\gamma+\frac{1-\gamma}{2 \gamma} \lambda_{r}(t)^{2} & -\frac{1}{2}\left(b(t)+\frac{1-\gamma}{\gamma} \sigma_{r}(t) \lambda_{r}(t)\right)
\end{array}\right) R, \\
& R(0)=\left(\begin{array}{c}
I_{m} \\
0_{m \times m}
\end{array}\right) .
\end{aligned}
$$

In particular, if $b(t), \sigma_{r}(t)$, and $\lambda_{r}(t)$ are constants, then

$$
\left.R(\tau)=\exp \left[\left(\begin{array}{cc}
\frac{1}{2}\left(b+\frac{1-\gamma}{\gamma} \sigma_{r} \lambda_{r}\right) & -\frac{\sigma_{r}^{2}}{2 \gamma} \\
1-\gamma+\frac{1-\gamma}{2 \gamma} \lambda_{r}^{2} & -\frac{1}{2}\left(b+\frac{1-\gamma}{\gamma} \sigma_{r} \lambda_{r}\right.
\end{array}\right)\right] \tau\right]\left(\begin{array}{c}
I_{m} \\
0_{m \times m}
\end{array}\right) .
$$


And, (2)

$$
\begin{gathered}
M(t, T)=\int_{t}^{T}\left[a(s) K(s, T)+\mathrm{E}\left[\left(e^{-z(1-\gamma)}-1\right) \mu(s)\right]\right. \\
\left.-\frac{1-\gamma}{2 \gamma}\|\Lambda(s)\|^{2}\right] d s,
\end{gathered}
$$

where $\mathrm{E}\left[e^{-z(1-\gamma)}\right]$ is the moment generating function of $z$.

Proof. The solution of the matrix Riccati differential equation (17) can be solved by the Radon lemma, a proof of which can be found in Theorem 3.1.1 of [13]. The Radon lemma immediately gives the explicit expression for $K(t, T) . M(t, T)$ is obtained by a simple and direct integration. Hence, the results follow.

3.2. Logarithmic Utility. When $\gamma$ approaches 1 , the power utility function tends to a logarithmic function. The corresponding investment policy and objective value require a separated analysis. We now concentrate on the maximization of the expected logarithmic utility function: $\mathrm{E}[U(Y(T))]=$ $\mathrm{E}[\ln Y(T)]$. Applying Itô's lemma to the log-wealth process with respect to (11),

$$
\begin{aligned}
& d(\ln Y)=\left(r+\pi^{\prime}\left(\begin{array}{c}
\Sigma_{s} \Lambda+\Sigma_{r} \lambda_{r} r \\
\sigma_{B} \lambda_{r} \sqrt{r}
\end{array}\right)-\frac{1}{2} \pi \Sigma \pi\right) d t \\
& +\pi^{\prime}\left(\begin{array}{cc}
\Sigma_{s} & \Sigma_{r} \sqrt{r} \\
\mathbf{0}_{1 \times n} & \sigma_{B}
\end{array}\right) d W_{t} \\
& +\left(\ln \left(Y-Y\left(1-e^{-z}\right)\right)-\ln Y\right) d \mathcal{N}_{t} \\
& =\left[r+\pi^{\prime}\left(\begin{array}{c}
\sum_{s} \Lambda+\Sigma_{r} \lambda_{r} r \\
\sigma_{B} \lambda_{r} \sqrt{r}
\end{array}\right)-\frac{1}{2} \pi \Sigma \pi-\mu z\right] d t \\
& +\pi^{\prime}\left(\begin{array}{cc}
\Sigma_{s} & \Sigma_{r} \sqrt{r} \\
\mathbf{0}_{1 \times n} & \sigma_{B}
\end{array}\right) d W_{t}-z d \mathscr{M}_{t} \\
& =\left\{r-\mu z+\frac{1}{2}\left(\begin{array}{c}
\Sigma_{s} \Lambda+\Sigma_{r} \lambda_{r} r \\
\sigma_{B} \lambda_{r} \sqrt{r}
\end{array}\right)^{\prime} \Sigma^{-1}\left(\begin{array}{c}
\Sigma_{s} \Lambda+\Sigma_{r} \lambda_{r} r \\
\sigma_{B} \lambda_{r} \sqrt{r}
\end{array}\right)\right. \\
& -\frac{1}{2}\left[\pi-\Sigma^{-1}\left(\begin{array}{c}
\Sigma_{s} \Lambda+\Sigma_{r} \lambda_{r} r \\
\sigma_{B} \lambda_{r} \sqrt{r}
\end{array}\right)\right]^{\prime} \\
& \left.\times \Sigma\left[\pi-\Sigma^{-1}\left(\begin{array}{c}
\Sigma_{s} \Lambda+\Sigma_{r} \lambda_{r} r \\
\sigma_{B} \lambda_{r} \sqrt{r}
\end{array}\right)\right]\right\} d t \\
& +\pi^{\prime}\left(\begin{array}{cc}
\Sigma_{s} & \Sigma_{r} \sqrt{r} \\
\mathbf{0}_{1 \times n} & \sigma_{B}
\end{array}\right) d W_{t}-z d \mathscr{M}_{t},
\end{aligned}
$$

where $\pi(t)=u(t) / Y(t)=(1 / Y(t))\left(\begin{array}{l}u_{s}(t) \\ u_{B}(t)\end{array}\right):=\left(\begin{array}{c}\pi_{s}(t) \\ \pi_{B}(t)\end{array}\right)$ is wealth portion invested in risky assets at time $t ; \Sigma=$ $\left(\begin{array}{cc}\Sigma_{s} \Sigma_{s}^{\prime}+\Sigma_{s} \Sigma_{s}^{\prime} r & \Sigma_{r} \sigma_{B} \sqrt{r} \\ \Sigma_{r}^{\prime} \sigma_{B} \sqrt{r} & \sigma_{B}^{2}\end{array}\right) ; \mathscr{M}_{t}:=\mathcal{N}_{t}-\int_{0}^{T} \mu(s) d s$ is a G-martingale. Hence, we have

$$
\begin{gathered}
\max _{\pi(\cdot)} \mathrm{E}[\ln Y(T)] \\
=\ln Y(0)
\end{gathered}
$$

$$
\begin{aligned}
+\max _{\pi(\cdot)} \mathrm{E}\left[\int_{0}^{T}\{\right. & r-\mu z+\frac{1}{2}\left(\begin{array}{c}
\Sigma_{s} \Lambda+\Sigma_{r} \lambda_{r} r \\
\sigma_{B} \lambda_{r} \sqrt{r}
\end{array}\right)^{\prime} \\
& \times \Sigma^{-1}\left(\begin{array}{c}
\Sigma_{s} \Lambda+\Sigma_{r} \lambda_{r} r \\
\sigma_{B} \lambda_{r} \sqrt{r}
\end{array}\right) \\
& -\frac{1}{2}\left[\pi-\Sigma^{-1}\left(\begin{array}{c}
\Sigma_{s} \Lambda+\Sigma_{r} \lambda_{r} r \\
\sigma_{B} \lambda_{r} \sqrt{r}
\end{array}\right)\right]^{\prime} \\
& \left.\left.\times \Sigma\left[\pi-\Sigma^{-1}\left(\begin{array}{c}
\Sigma_{s} \Lambda+\Sigma_{r} \lambda_{r} r \\
\sigma_{B} \lambda_{r} \sqrt{r}
\end{array}\right)\right]\right\} d t\right]
\end{aligned}
$$

It is thus clear that the expected final utility attains its maximum value at

$$
\begin{aligned}
\pi^{*} & =\left(\begin{array}{c}
\pi_{s}^{*} \\
\pi_{B}^{*}
\end{array}\right) \\
& =\Sigma^{-1}\left(\begin{array}{c}
\Sigma_{s} \Lambda+\Sigma_{r} \lambda_{r} r \\
\sigma_{B} \lambda_{r} \sqrt{r}
\end{array}\right) \\
& =\left(\begin{array}{c}
\left(\Sigma_{s}^{\prime}\right)^{-1} \Lambda \\
\frac{\sqrt{r}}{\sigma_{B}}\left(\lambda_{r}-\Lambda^{\prime} \Sigma_{s}^{-1} \Sigma_{r}\right)
\end{array}\right),
\end{aligned}
$$

and the maximum objective value is

$$
\begin{aligned}
& \ln Y(0)+\int_{0}^{T} r(t) d t-\mathrm{E}[z] \int_{0}^{T} \mathrm{E}[\mu(t)] d t \\
& +\frac{1}{2} \mathrm{E}\left[\int_{0}^{T}\left(\begin{array}{c}
\Sigma_{s}(t) \Lambda(t)+\Sigma_{r}(t) \lambda_{r}(t) r(t) \\
\sigma_{B}(t) \lambda_{r}(t) \sqrt{r(t)}
\end{array}\right)\right. \\
& \left.\quad \times \Sigma^{-1}(t)\left(\begin{array}{c}
\Sigma_{s}(t) \Lambda(t)+\Sigma_{r}(t) \lambda_{r}(t) r(t) \\
\sigma_{B}(t) \lambda_{r}(t) \sqrt{r(t)}
\end{array}\right) d t\right] .
\end{aligned}
$$

Theorem 6. Under Assumptions 1 and 2, the research problem (13) with the logarithmic utility (14) has the optimal investment policy:

$$
\begin{aligned}
u^{*}(t, Y(t), r(t)) & =\left(\begin{array}{l}
u_{s}^{*} \\
u_{B}^{*}
\end{array}\right) \\
& =\left(\begin{array}{c}
\left(\Sigma_{s}^{\prime}\right)^{-1} \Lambda \\
\frac{\sqrt{r}}{\sigma_{B}}\left(\lambda_{r}-\Lambda^{\prime} \Sigma_{s}^{-1} \Sigma_{r}\right)
\end{array}\right) Y(t),
\end{aligned}
$$

and the optimal value function, $\left.\mathrm{E}[\ln Y(T)]\right|_{\mathcal{u}=u^{*}}$, equals

$$
\begin{aligned}
\ln Y_{0}+ & \int_{0}^{T} \frac{\|\Lambda(s)\|^{2}}{2} d s-\mathrm{E}[z] \int_{0}^{T} \mathrm{E}[\mu(s)] d s \\
+ & \int_{0}^{T}\left(e^{-\int_{0}^{s} b(\tau) d \tau} r(0)+\int_{0}^{s} e^{-\int_{\tau}^{s} b(\eta) d \eta} a(\tau) d \tau\right) \\
& \times\left(1-\frac{\lambda_{r}(s)^{2}}{2}\right) d s .
\end{aligned}
$$


Proof. From the analysis prior to this theorem, we have already shown (33), which is equivalent to (35). It remains for us to simplify

$$
\begin{aligned}
& \mathrm{E}\left[\int_{0}^{T} r(t)+\frac{1}{2}\left(\begin{array}{c}
\Sigma_{s}(t) \Lambda(t)+\Sigma_{r}(t) \lambda_{r}(t) r(t) \\
\sigma_{B}(t) \lambda_{r}(t) \sqrt{r(t)}
\end{array}\right)^{\prime}\right. \\
& \left.\quad \times \Sigma^{-1}(t)\left(\begin{array}{c}
\Sigma_{s}(t) \Lambda(t)+\Sigma_{r}(t) \lambda_{r}(t) r(t) \\
\sigma_{B}(t) \lambda_{r}(t) \sqrt{r(t)}
\end{array}\right) d t\right] .
\end{aligned}
$$

According to (23),

$$
\Sigma^{-1}=\left(\begin{array}{cc}
\left(\Sigma_{s} \Sigma_{s}^{\prime}\right)^{-1} & -\frac{\sqrt{r}}{\sigma_{B}}\left(\Sigma_{s} \Sigma_{s}^{\prime}\right)^{-1} \Sigma_{r} \\
-\frac{\sqrt{r}}{\sigma_{B}} \Sigma_{r}^{\prime}\left(\Sigma_{s} \Sigma_{s}^{\prime}\right)^{-1} & \frac{1+r \Sigma_{r}^{\prime}\left(\Sigma_{s} \Sigma_{s}^{\prime}\right)^{-1} \Sigma_{r}}{\sigma_{B}^{2}}
\end{array}\right) .
$$

Hence,

$$
\begin{aligned}
r+ & \frac{1}{2}\left(\begin{array}{c}
\Sigma_{s} \Lambda+\Sigma_{r} \lambda_{r} r \\
\sigma_{B} \lambda_{r} \sqrt{r}
\end{array}\right)^{\prime} \Sigma^{-1}\left(\begin{array}{c}
\Sigma_{s} \Lambda+\Sigma_{r} \lambda_{r} r \\
\sigma_{B} \lambda_{r} \sqrt{r}
\end{array}\right) \\
= & r+\frac{1}{2}\left(\begin{array}{c}
\Sigma_{s} \Lambda+\Sigma_{r} \lambda_{r} r \\
\sigma_{B} \lambda_{r} \sqrt{r}
\end{array}\right)^{\prime} \\
& \times\left(\begin{array}{c}
\left(\Sigma_{s} \Sigma_{s}^{\prime}\right)^{-1}-\frac{\sqrt{r}}{\sigma_{B}}\left(\Sigma_{s} \Sigma_{s}^{\prime}\right)^{-1} \Sigma_{r} \\
-\frac{\sqrt{r}}{\sigma_{B}} \Sigma_{r}^{\prime}\left(\Sigma_{s} \Sigma_{s}^{\prime}\right)^{-1} \frac{1+r \Sigma_{r}^{\prime}\left(\Sigma_{s} \Sigma_{s}^{\prime}\right)^{-1} \Sigma_{r}}{\sigma_{B}^{2}}
\end{array}\right) \\
& \times\left(\begin{array}{c}
\Sigma_{s} \Lambda+\Sigma_{r} \lambda_{r} r \\
\sigma_{B} \lambda_{r} \sqrt{r}
\end{array}\right) \\
= & +\frac{1}{2}\left(\begin{array}{c}
\Sigma_{s} \Lambda+\Sigma_{r} \lambda_{r} r \\
\sigma_{B} \lambda_{r} \sqrt{r}
\end{array}\right)^{\prime} \\
& \times\left(\begin{array}{c}
\left(\Sigma_{s}^{\prime}\right)^{-1} \Lambda \\
-\frac{\sqrt{r}}{\sigma_{B}} \Lambda^{\prime} \Sigma_{s}^{-1} \Sigma_{r}-\frac{1}{\sigma_{B}} \lambda_{r} \sqrt{r}
\end{array}\right) \\
= & +\frac{\|\Lambda\|^{2}}{2}-\frac{r \lambda_{r}^{2}}{2}=\frac{\|\Lambda\|^{2}}{2}+r\left(1-\frac{\lambda_{r}^{2}}{2}\right) .
\end{aligned}
$$

According to the interest rate dynamic (2), it is easy to derive that

$$
\mathrm{E}[r(s)]=e^{-\int_{0}^{s} b(\tau) d \tau} r(0)+\int_{0}^{s} e^{-\int_{\tau}^{s} b(\eta) d \eta} a(\tau) d \tau
$$

Substituting (39) and (40) into (34) verifies that the optimal value function equals (36).

3.3. Verification Theorem. The following two propositions together serve as a verification theorem for the solution of the $\mathrm{HJB}$ equation in (20). The results and proofs are classical. We adopt the framework of [10]. For smoothening the proofs of the propositions, a notation is introduced as follows:

$$
\begin{aligned}
\mathscr{L}^{u} J= & \frac{\partial J}{\partial t}+\frac{\partial J}{\partial r}(a-b r)+\frac{\partial J}{\partial y}\left(r y+u^{\prime}\left(\begin{array}{c}
\Sigma_{s} \Lambda+\Sigma_{r} \lambda_{r} r \\
\lambda_{r} \sqrt{r}
\end{array}\right)\right) \\
& +\frac{1}{2} \frac{\partial^{2} J}{\partial r^{2}} \sigma_{r}^{2} r+\frac{1}{2} \frac{\partial^{2} J}{\partial y^{2}} u^{\prime}\left(\begin{array}{cc}
\Sigma_{s} \Sigma_{s}^{\prime}+\Sigma_{s} \Sigma_{s}^{\prime} r & \Sigma_{r} \sigma_{B} \sqrt{r} \\
\Sigma_{r}^{\prime} \sigma_{B} \sqrt{r} & \sigma_{B}^{2}
\end{array}\right) u \\
& -\frac{\partial^{2} J}{\partial r \partial y} \sigma_{r} \sqrt{r} u^{\prime}\left(\begin{array}{c}
\Sigma_{r} \sqrt{r} \\
\sigma_{B}
\end{array}\right) \\
& +\mathrm{E}\left[\left(J\left(t, y-y\left(1-e^{-z}\right), r\right)-J(t, y, r)\right) \mu\right] .
\end{aligned}
$$

Clearly, the HJB equation in (20) can be rewritten as $\sup _{u} \mathscr{L}^{u} V=0$.

Proposition 7. It is assumed that $J \in C^{2}\left([0, T] \times \mathbb{R}_{+}^{2}\right)$ is a nonnegative function such that $\mathscr{L}^{u} J \leq 0, J(T, y, r)=U(y)$, and $J(t, 0, r)=0$ for every admissible control $u$. Then

$$
J(t, y, r) \geq E\left[U\left(Y^{u}(T)\right) \mid \mathscr{G}_{t}\right]
$$

Proof. It is assumed that $u$ is an admissible control, and let $\left\{\tau_{n}\right\}_{n}$ be a localizing sequence of stopping times for the local semimartingale $\left(Y^{u}(s), r(s)\right)$ starting with $(y, r)$ at time $t$. Applying Itô's lemma to $J$ with respect to (11), we have

$$
\begin{aligned}
& d J=\left\{\frac{\partial J}{\partial t}+\frac{\partial J}{\partial r}(a-b r)\right. \\
& +\frac{\partial J}{\partial y}\left(r y+u^{\prime}\left(\begin{array}{c}
\Sigma_{s} \Lambda+\Sigma_{r} \lambda_{r} r \\
\lambda_{r} \sqrt{r}
\end{array}\right)\right)+\frac{1}{2} \frac{\partial^{2} J}{\partial r^{2}} \sigma_{r}^{2} r \\
& +\frac{1}{2} \frac{\partial^{2} J}{\partial y^{2}} u^{\prime}\left(\begin{array}{cc}
\Sigma_{s} \Sigma_{s}^{\prime}+\Sigma_{s} \Sigma_{s}^{\prime} r & \Sigma_{r} \sigma_{B} \sqrt{r} \\
\Sigma_{r}^{\prime} \sigma_{B} \sqrt{r} & \sigma_{B}^{2}
\end{array}\right) u \\
& \left.-\frac{\partial^{2} J}{\partial r \partial y} \sigma_{r} \sqrt{r} u^{\prime}\left(\begin{array}{c}
\Sigma_{r} \sqrt{r} \\
\sigma_{B}
\end{array}\right)\right\} d t \\
& +\left\{\frac{\partial J}{\partial y} u^{\prime}\left(\begin{array}{cc}
\Sigma_{s} & \Sigma_{r} \sqrt{r} \\
\mathbf{0}_{1 \times n} & \sigma_{B}
\end{array}\right)-\frac{\partial J}{\partial r}\left(\begin{array}{ll}
\mathbf{0}_{1 \times n} & \sigma_{r} \sqrt{r}
\end{array}\right)\right\} d W_{t} \\
& +\left(J\left(t, y-y\left(1-e^{-z}\right), r\right)-J(t, y, r)\right) d \mathcal{N}_{t} \\
& =\left\{\frac{\partial J}{\partial t}+\frac{\partial J}{\partial r}(a-b r)\right. \\
& +\frac{\partial J}{\partial y}\left(r y+u^{\prime}\left(\begin{array}{c}
\Sigma_{s} \Lambda+\Sigma_{r} \lambda_{r} r \\
\lambda_{r} \sqrt{r}
\end{array}\right)\right)+\frac{1}{2} \frac{\partial^{2} J}{\partial r^{2}} \sigma_{r}^{2} r \\
& +\frac{1}{2} \frac{\partial^{2} J}{\partial y^{2}} u^{\prime}\left(\begin{array}{cc}
\Sigma_{s} \Sigma_{s}^{\prime}+\Sigma_{s} \Sigma_{s}^{\prime} r & \Sigma_{r} \sigma_{B} \sqrt{r} \\
\Sigma_{r}^{\prime} \sigma_{B} \sqrt{r} & \sigma_{B}^{2}
\end{array}\right) u \\
& \left.-\frac{\partial^{2} J}{\partial r \partial y} \sigma_{r} \sqrt{r} u^{\prime}\left(\begin{array}{c}
\Sigma_{r} \sqrt{r} \\
\sigma_{B}
\end{array}\right)\right\} d t
\end{aligned}
$$




$$
\begin{aligned}
& +\left(J\left(t, y-y\left(1-e^{-z}\right), r\right)-J(t, y, r)\right) \mu(t) d t
\end{aligned}
$$

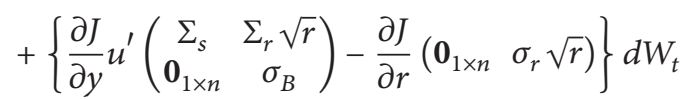

$$
\begin{aligned}
& +\left(J\left(t, y-y\left(1-e^{-z}\right), r\right)-J(t, y, r)\right) d \mathscr{M}_{t} .
\end{aligned}
$$

Hence, we have

$$
\begin{aligned}
\mathrm{E} & {\left[J\left(T \wedge \tau_{n}, Y^{u}\left(T \wedge \tau_{n}\right), r\left(T \wedge \tau_{n}\right)\right) \mid \mathscr{G}_{t}\right] } \\
& =J(t, y, r)+\int_{t}^{T \wedge \tau_{n}} \mathscr{L}^{u} J\left(s, Y^{u}(s), r(s)\right) d s \\
& \leq J(t, y, r) .
\end{aligned}
$$

Because of the nonnegativity of $J,\left\{J\left(T \wedge \tau_{n}, Y^{u}\left(T \wedge \tau_{n}\right), r(T \wedge\right.\right.$ $\left.\left.\left.\tau_{n}\right)\right)\right\}_{n}$ is a sequence of nonnegative measurable functions. Since

$$
\begin{aligned}
& \lim _{n \rightarrow \infty} J\left(T \wedge \tau_{n}, Y^{u}\left(T \wedge \tau_{n}\right), r\left(T \wedge \tau_{n}\right)\right) \\
&=J\left(T, Y^{u}(T), r(T)\right)=U\left(Y^{u}(T)\right), \quad \text { a.s., }
\end{aligned}
$$

Fatou's lemma yields

$$
\begin{aligned}
\mathrm{E} & {\left[U\left(Y^{u}(T)\right) \mid \mathscr{G}_{t}\right] } \\
& =\mathrm{E}\left[\liminf _{n \rightarrow \infty} J\left(T \wedge \tau_{n}, Y^{u}\left(T \wedge \tau_{n}\right), r\left(T \wedge \tau_{n}\right)\right) \mid \mathscr{G}_{t}\right] \\
& \leq \liminf _{n \rightarrow \infty} \mathrm{E}\left[J\left(T \wedge \tau_{n}, Y^{u}\left(T \wedge \tau_{n}\right), r\left(T \wedge \tau_{n}\right)\right) \mid \mathscr{G}_{t}\right] \\
& \leq J(t, y, r) .
\end{aligned}
$$

Proposition 8. Let $J \in C^{2}\left([0, T] \times \mathbb{R}_{+}^{2}\right)$ be a nonnegative function such that the family of random variables $\left\{J\left(\tau, Y^{u^{*}}(\tau), r(\tau)\right)\right\}_{\tau}$ is uniformly integrable, where $u^{*}$ is an admissible control with the property $\mathscr{L}^{u^{*}} J=0$ and $\tau \in[t, T]$ is a stopping time for the process $\left(Y^{u^{*}}(s), r(s)\right)$ starting with $(y, r)$ at time $t$. If, furthermore, $J(T, y, r)=U(y), J(t, 0, r)=0$, and $\mathscr{L}^{u} J \leq 0$ for all admissible controls $u$, then

$$
J(t, y, r)=V(t, y, r) \quad \forall(t, y, r) \in[0, T] \times \mathbb{R}_{+}^{2} .
$$

Proof. Thanks to the uniform integrability of the family $\left\{J \tau,\left(Y^{u^{*}}(\tau), r(\tau)\right)\right\}_{\tau}$, we have

$$
\begin{aligned}
\mathrm{E} & {\left[U\left(Y^{u^{*}}(T)\right) \mid \mathscr{G}_{t}\right] } \\
& =\mathrm{E}\left[\lim _{n \rightarrow \infty} J\left(T \wedge \tau_{n}, Y^{u^{*}}\left(T \wedge \tau_{n}\right), r\left(T \wedge \tau_{n}\right)\right) \mid \mathscr{G}_{t}\right] \\
& =\lim _{n \rightarrow \infty} \mathrm{E}\left[J\left(T \wedge \tau_{n}, Y^{u^{*}}\left(T \wedge \tau_{n}\right), r\left(T \wedge \tau_{n}\right)\right) \mid \mathscr{G}_{t}\right] \\
& \leq J(t, y, r) .
\end{aligned}
$$

Furthermore, $\mathscr{L}^{u^{*}} J=0$ induce that $\mathrm{E}\left[U\left(Y^{u^{*}}(T)\right) \mid \mathscr{G}_{t}\right]=$ $J(t, y, r)$. Hence, by Proposition $7, J(t, y, r)=V(t, y, r)$ for all $(t, y, r) \in[0, T] \times \mathbb{R}_{+}^{2}$.

The above propositions are classical and valid for a class of positive utility functions including the CRRA utility. To verify the uniform integrability of $\left\{J\left(\tau, Y^{u^{*}}(\tau), r(\tau)\right)\right\}_{\tau}$, we need to calculate $\mathrm{E}\left[J\left(\tau, Y^{u^{*}}(\tau), r(\tau)\right) \mid \mathscr{G}_{t}\right]$, where

$$
\begin{aligned}
& J(\tau, Y(\tau), r(\tau)) \\
& \qquad \begin{array}{cc}
V(\tau, Y(\tau), r(\tau)) & \text { when } U(y)=\frac{y^{1-\gamma}}{1-\gamma} \\
\ln Y(\tau)+\int_{\tau}^{T} \frac{\|\Lambda(s)\|^{2}}{2} d s & \\
-\mathrm{E}[z] \int_{\tau}^{T} \mathrm{E}[\mu(s)] d s & \\
+\int_{\tau}^{T}\left(e^{-\int_{\tau}^{s} b(\eta) d \eta} r(\tau)\right. & \\
\left.+\int_{\tau}^{s} e^{-\int_{\eta}^{s} b(\theta) d \theta} a(\eta) d \eta\right) & \text { when } U(y)=\ln y ; \\
\times\left(1-\frac{\lambda_{r}(s)^{2}}{2}\right) d s \quad
\end{array}
\end{aligned}
$$

$V(t, Y(t), r(\tau))$ is given in (26). The determination of the positivity of $K$ (the solution of the Riccati differential equation (17)) will be discussed. The following proposition is a comparison theorem for the solutions of standard Riccati differential equations. The details can be found in [14].

Proposition 9. For $i=1,2$, let $K_{i}$ be the solution of

$$
\dot{K}_{i}=-A_{i}^{\prime}(t) K_{i}-K_{i} A_{i}(t)-Q_{i}(t)+K_{i} S_{i}(t) K_{i}
$$

on some interval $\mathscr{I}$. If for some $t_{f} \in \mathscr{I}, K_{1}\left(t_{f}\right) \leq K_{2}\left(t_{f}\right)$ and if

$$
\left(\begin{array}{cc}
Q_{2} & A_{2}^{\prime} \\
A_{2} & -S_{2}
\end{array}\right)(t)-\left(\begin{array}{cc}
Q_{1} & A_{1}^{\prime} \\
A_{1} & -S_{1}
\end{array}\right)(t) \geq \mathbf{0} \quad \text { for } t \in \mathscr{I},
$$

where 0 is a matrix with zero valued entries, then $K_{1}(t) \leq K_{2}(t)$ for all $t \in \mathscr{I} \cap\left(-\infty, t_{f}\right]$.

Lemma 10. The solution of $O D E(17), K(t, T)$, is a nonnegative function of time $t$ for all $t \in[0, T]$.

Proof. Consider $A_{1}=A_{2}=-(1 / 2)\left(b+((1-\gamma) / \gamma) \sigma_{r} \lambda_{r}\right), S_{1}=$ $S_{2}=-\sigma_{r}^{2} / 2 \gamma<0, Q_{1}=0$, and $Q_{2}=\left(1-\gamma+((1-\gamma) / 2 \gamma) \lambda_{r}^{2}\right)>0$. It is obvious that $0_{m \times m}$ is the solution of

$$
\begin{aligned}
\dot{K}_{1}= & -A_{1}^{\prime}(t) K_{1}-K_{1} A_{1}(t) \\
& -Q_{1}(t)+K_{1} S_{1}(t) K_{1}, \quad K_{1}(T)=0 ;
\end{aligned}
$$

and the solution of the ODE (17), $K(t, T)$, is the solution of

$$
\begin{aligned}
\dot{K}_{2}= & -A_{2}^{\prime}(t) K_{2}-K_{2} A_{2}(t) \\
& -Q_{2}(t)+K_{2} S_{2}(t) K_{2}, \quad K_{2}(T)=0 .
\end{aligned}
$$


Therefore, we have

$$
\begin{aligned}
& \left(\begin{array}{cc}
Q_{2} & A_{2}^{\prime} \\
A_{2} & -S_{2}
\end{array}\right)(t)-\left(\begin{array}{cc}
Q_{1} & A_{1}^{\prime} \\
A_{1} & -S_{1}
\end{array}\right)(t) \\
& \quad=\left(\begin{array}{cc}
1-\gamma+\frac{1-\gamma}{2 \gamma} \lambda_{r}^{2} & 0 \\
0 & 0
\end{array}\right)(t) \geq \mathbf{0}_{2 \times 2}
\end{aligned}
$$

and $K_{2}(T) \geq K_{1}(T)$. Applying Proposition 9, $K(t, T)=$ $K_{2}(t) \geq 0$ for all $t \in[0, T]$, which means that the solution of the matrix $\operatorname{RDE}(17), K(t, T)$, is a semipositive definite matrix for all $t \in[0, T]$.

Next step is a calculation of the expected value of $J\left(\tau, Y^{u^{*}}(\tau), r(\tau)\right)$ at filtration $\mathscr{G}_{t}$, where $0 \leq t \leq \tau$.

Lemma 11. Given $0 \leq t \leq \tau \leq T$ and using the notations in Theorem 4,

$$
\begin{aligned}
\mathrm{E}\left[V\left(\tau, Y^{u^{*}}(\tau), r(\tau)\right) \mid \mathscr{G}_{t}\right] \\
=\mathrm{E}\left[\frac{\left(Y^{u^{*}}(\tau)\right)^{1-\gamma}}{1-\gamma} e^{K(\tau, T) r(\tau)+M(\tau, T)} \mid \mathscr{G}_{t}\right] \\
=\frac{(Y(t))^{1-\gamma} \psi_{\mu^{*}(t, \tau)}\left(\psi_{z}(1-\gamma)-1\right)}{1-\gamma} e^{\widetilde{K}(t, \tau) r(t)+\widetilde{M}(t, \tau)},
\end{aligned}
$$

where $\widetilde{K}(t, \tau)$ and $\widetilde{M}(t, \tau)$ satisfy the system of ordinary differential equations (ODEs):

$$
\begin{gathered}
\dot{\widetilde{K}}-\left(b+\frac{1-\gamma}{\gamma}\left(\lambda_{r} \sigma_{r}-K \sigma_{r}^{2}\right)\right) \\
+\frac{\sigma_{r}^{2}}{2} \widetilde{K}^{2}+1-\gamma+\frac{1-\gamma}{2 \gamma}\left(\lambda_{r}^{2}-K^{2} \sigma_{r}^{2}\right)=0, \\
\widetilde{K}(\tau, \tau)=K(\tau, T) ; \\
\dot{\bar{M}}+\widetilde{K} a+\frac{1-\gamma}{2 \gamma}\|\Lambda\|^{2}=0, \\
\widetilde{M}(\tau, \tau)=M(\tau, T)
\end{gathered}
$$

$\psi_{\mathscr{E}}(t)$ is a moment generating function of random variable $\mathscr{Z}$ and $\mu^{*}(t, \tau)=\int_{t}^{\tau} \mu(s) d s$.

Proof. Let

$$
\begin{aligned}
& \widetilde{v}(t, Y, r) \\
& \quad=\frac{Y^{1-\gamma} \psi_{\mu^{*}(t, \tau)}\left(\psi_{z}(1-\gamma)-1\right)}{1-\gamma} e^{\widetilde{K}(t, \tau) r+\widetilde{M}(t, \tau)},
\end{aligned}
$$

where $\widetilde{K}$ and $\widetilde{M}$ are the solution of the system of ODEs (56) and (57), respectively; $r$ follows the dynamic:

$$
d r(t)=(a-b r) d t-\sigma_{r} \sqrt{r} d W_{t}^{r}
$$

and $Y^{*}$ follows the dynamic:

$$
\begin{aligned}
& \frac{d Y}{Y}=r^{*} d t+\sigma^{*} d W_{t}-\left(1-e^{-z}\right) d \mathcal{N}_{t}, \\
& r^{*}=r\left(1+\frac{1+\lambda_{r}^{2}-\lambda_{r} K \sigma_{r}}{\gamma}\right)+\frac{\|\Lambda\|^{2}}{\gamma}, \\
& \sigma^{*}=\frac{1}{\gamma}\left(\Lambda^{\prime} \sqrt{r}\left(\lambda_{r}-K \sigma_{r}\right)\right) ;
\end{aligned}
$$

$\psi_{\mathscr{L}}(t)$ is a moment generating function of random variable $\mathscr{Z}$ and $\mu^{*}(t, \tau)=\int_{t}^{\tau} \mu(s) d s$. Clearly,

$$
\begin{aligned}
\widetilde{v}(\tau, Y, r) & =\frac{Y^{1-\gamma}}{1-\gamma} e^{K(\tau, T) r+M(\tau, T)} \\
& =\mathrm{E}\left[\frac{Y(\tau)^{1-\gamma}}{1-\gamma} e^{K(\tau, T) r(\tau)+M(\tau, T)} \mid \mathscr{G}_{\tau}\right] \\
& =\mathrm{E}\left[V\left(\tau, Y^{u^{*}}(\tau), r(\tau)\right) \mid \mathscr{G}_{\tau}\right] .
\end{aligned}
$$

Next,

$$
\begin{aligned}
\widetilde{v}_{t}+\widetilde{v}_{r}(a-b r)+\frac{1}{2} \widetilde{v}_{r r} \sigma_{r}^{2} r \\
+\mathrm{E}\left[\left(\widetilde{v}\left(t, Y-Y\left(1-e^{-z}\right), r\right)-\widetilde{v}(t, Y, r)\right) \mu\right] \\
+\widetilde{v}_{Y}\left(r^{*} Y\right)+\frac{1}{2} \widetilde{v}_{Y Y} \sigma^{*} \sigma^{* \prime} Y^{2} \\
-\widetilde{v}_{r Y}\left(\frac{r}{\gamma} \sigma_{r}\left(\lambda_{r}-K \sigma_{r}\right)\right) Y
\end{aligned}
$$

would be calculated. Taking partial derivatives to $\widetilde{v}$ with respect to $t, Y$, and $r$, we have

$$
\begin{gathered}
\widetilde{v}_{t}=\left(-\mu\left(\psi_{z}(1-\gamma)-1\right)+\dot{\widetilde{K}} r+\dot{\bar{M}}\right) \widetilde{v} \\
=\left(-\mathrm{E}\left[\mu\left(e^{-(1-\gamma) z}-1\right)\right]+\dot{\widetilde{K}}^{\prime} r+\dot{M}\right) \widetilde{v} \\
\widetilde{v}_{r}=\widetilde{K} \widetilde{v} ; \quad \widetilde{v}_{r r}=\widetilde{K}^{2} \widetilde{v} ; \\
\widetilde{v}_{y}=\frac{1-\gamma_{\widetilde{v}} ; \quad \widetilde{v}_{y y}=-\frac{\gamma(1-\gamma)}{y^{2}} \widetilde{v} ;}{\widetilde{v}_{r y}=\frac{1-\gamma}{y} \widetilde{K} \widetilde{v} ;} \\
\mathrm{E}\left[\left(\widetilde{v}\left(t, y-y\left(1-e^{-z}\right), r\right)-\widetilde{v}\right) \mu\right] \\
=\mathrm{E}\left[\left(e^{-z(1-\gamma)}-1\right) \mu\right] \widetilde{v} .
\end{gathered}
$$


Substituting these expressions into (62) yields

$$
\begin{aligned}
\widetilde{v}_{t}+\widetilde{v}_{r}(a-b r)+\frac{1}{2} \widetilde{v}_{r r} \sigma_{r}^{2} r \\
+\mathrm{E}\left[\left(\widetilde{v}\left(t, Y-Y\left(1-e^{-z}\right), r\right)-\widetilde{v}(t, Y, r)\right) \mu\right] \\
+\widetilde{v}_{Y}\left(r^{*} Y\right)+\frac{1}{2} \widetilde{v}_{Y Y} \sigma^{*} \sigma^{* \prime} Y^{2} \\
-\widetilde{v}_{r Y}\left(\frac{r}{\gamma} \sigma_{r}\left(\lambda_{r}-K \sigma_{r}\right)\right) Y \\
=\widetilde{v}\left\{\left[\dot{\bar{K}}-\left(b+\frac{1-\gamma}{\gamma}\left(\lambda_{r} \sigma_{r}-K \sigma_{r}^{2}\right)\right)\right.\right. \\
\left.+\frac{\sigma_{r}^{2}}{2} \widetilde{K}^{2}+1-\gamma+\frac{1-\gamma}{2 \gamma}\left(\lambda_{r}^{2}-K^{2} \sigma_{r}^{2}\right)\right] r \\
\left.+\dot{\bar{M}}+\widetilde{K} a+\frac{1-\gamma}{2 \gamma}\|\Lambda\|^{2}\right\}=0 .
\end{aligned}
$$

Combining the equalities (61) and (64), $\widetilde{v}(t, Y, r)=$ $\mathrm{E}\left[V\left(\tau, Y^{u^{*}}(\tau), r(\tau)\right) \mid \mathscr{G}_{t}\right]$ is proved.

To show the uniform integrability of $\left\{V\left(\tau, Y^{u^{*}}(\tau), r(\tau)\right)\right\}_{\tau}$ is equivalent to showing the boundedness of the function value $\widetilde{v}(t, Y, r)$ by Lemma 11 . Hence, the boundedness of the solution of ODE in (56), $\widetilde{K}(t, \tau)$, induces the boundedness of function value $\widetilde{v}(t, Y, r)$. The following proposition (c.f. Theorem 4.3 of [15]) is useful for showing the boundedness of $\widetilde{K}(t, \tau)$.

Lemma 12. Given $0 \leq t \leq \tau \leq T$ and using the notations in Theorem 4 and Lemma 11,

$$
\widetilde{K}(t, \tau) \leq K(t, T),
$$

for any stopping times $\tau \in[0, T]$ and $t \in[0, \tau]$.

Proof. Consider a stopping time $\tau$ such that $\tau \in[0, T]$ and $t \in[0, \tau]$. By choosing

$$
\begin{gathered}
A_{1}=-\frac{1}{2}\left(b+\frac{1-\gamma}{\gamma}\left(\lambda_{r} \sigma_{r}-K \sigma_{r}^{2}\right)\right) ; \\
A_{2}=-\frac{1}{2}\left(b+\frac{1-\gamma}{\gamma} \sigma_{r} \lambda_{r}\right) ; \\
S_{1}=-\frac{\sigma_{r}^{2}}{2} ; \quad S_{2}=-\frac{\sigma_{r}^{2}}{2 \gamma} ; \\
Q_{1}=1-\gamma+\frac{1-\gamma}{2 \gamma}\left(\lambda_{r}^{2}-K^{2} \sigma_{r}^{2}\right) ; \\
Q_{2}=1-\gamma+\frac{1-\gamma}{2 \gamma} \lambda_{r}^{2}
\end{gathered}
$$

it is obvious that $\widetilde{K}(t, \tau)$ is the solution of

$$
\begin{array}{r}
\dot{K}_{1}=-A_{1}^{\prime}(t) K_{1}-K_{1} A_{1}(t)-Q_{1}(t)+K_{1} S_{1}(t) K_{1}, \\
K_{1}(\tau)=\widetilde{K}(\tau, \tau) ;
\end{array}
$$

and the solution of the matrix $\operatorname{RDE}(17), K(t, T)$, is the solution of

$$
\begin{array}{r}
\dot{K}_{2}=-A_{2}^{\prime}(t) K_{2}-K_{2} A_{2}(t)-Q_{2}(t)+K_{2} S_{2}(t) K_{2}, \\
K_{2}(\tau)=K(\tau, T) .
\end{array}
$$

Therefore, we have

$$
\begin{gathered}
\left(\begin{array}{cc}
Q_{2} & A_{2}^{\prime} \\
A_{2} & -S_{2}
\end{array}\right)(t)-\left(\begin{array}{cc}
Q_{1} & A_{1}^{\prime} \\
A_{1} & -S_{1}
\end{array}\right)(t) \\
=\frac{1-\gamma}{2 \gamma}\left(\begin{array}{cc}
K^{2} \sigma_{r}^{2} & -K \sigma_{r}^{2} \\
-K \sigma_{r}^{2} & \sigma_{r}^{2}
\end{array}\right)(t) .
\end{gathered}
$$

Clearly, $K^{2} \sigma_{r}^{2}$ and $(1-\gamma) / 2 \gamma$ are greater than zero; and

$$
\operatorname{det}\left(\begin{array}{cc}
K^{2} \sigma_{r}^{2} & -K \sigma_{r}^{2} \\
-K \sigma_{r}^{2} & \sigma_{r}^{2}
\end{array}\right)=0
$$

Hence, the matrix in (69), $((1-\gamma) / 2 \gamma)\left(\begin{array}{cc}K^{2} \sigma_{r}^{2} & -K \sigma_{r}^{2} \\ -K \sigma_{r}^{2} & \sigma_{r}^{2}\end{array}\right)(t)$, is semipositive definite. Combining the fact that $K(\tau, T)=$ $K_{2}(\tau) \geq K_{1}(\tau)=\widetilde{K}(\tau, \tau)$ and applying Proposition 9, $\widetilde{K}(t, \tau) \geq K(t, T)$ for all $t \in[0, \tau]$.

Lemma 13. Given $0 \leq t \leq \tau \leq T$ and using the notations in Theorem 4 and Lemma 11,

$$
k(t, \tau) \leq \widetilde{K}(t, \tau),
$$

for any stopping times $\tau \in[0, T]$ and $t \in[0, \tau]$, where $k(t, \tau)$ is the non-blow-up solution of the following RDE:

$$
\begin{gathered}
\dot{k}-\left(b+\frac{1-\gamma}{\gamma}\left(\lambda_{r} \sigma_{r}-K \sigma_{r}^{2}\right)\right) \\
+\frac{\sigma_{r}^{2}}{2} k^{2}-\frac{1-\gamma}{2 \gamma} K^{2} \sigma_{r}^{2}=0, \\
k(\tau, \tau)=K(\tau, T) .
\end{gathered}
$$

Proof. Consider a stopping time $\tau$ such that $\tau \in[0, T]$ and $t \in[0, \tau]$. By choosing

$$
\begin{gathered}
A_{1}=-\frac{1}{2}\left(b+\frac{1-\gamma}{\gamma}\left(\lambda_{r} \sigma_{r}-K \sigma_{r}^{2}\right)\right) ; \\
A_{2}=-\frac{1}{2}\left(b+\frac{1-\gamma}{\gamma}\left(\lambda_{r} \sigma_{r}-K \sigma_{r}^{2}\right)\right) ; \\
S_{1}=-\frac{\sigma_{r}^{2}}{2} ; \quad S_{2}=-\frac{\sigma_{r}^{2}}{2} ; \\
Q_{1}=-\frac{1-\gamma}{2 \gamma} K^{2} \sigma_{r}^{2} ; \\
Q_{2}=1-\gamma+\frac{1-\gamma}{2 \gamma}\left(\lambda_{r}^{2}-K^{2} \sigma_{r}^{2}\right) ;
\end{gathered}
$$


it is obvious that $k(t, \tau)$ is the solution of

$$
\begin{array}{r}
\dot{K}_{1}=-A_{1}^{\prime}(t) K_{1}-K_{1} A_{1}(t)-Q_{1}(t)+K_{1} S_{1}(t) K_{1}, \\
K_{1}(\tau)=k(\tau, \tau) ;
\end{array}
$$

and $\widetilde{K}(t, \tau)$ is the solution of

$$
\begin{array}{r}
\dot{K}_{2}=-A_{2}^{\prime}(t) K_{2}-K_{2} A_{2}(t)-Q_{2}(t)+K_{2} S_{2}(t) K_{2}, \\
K_{2}(\tau)=\widetilde{K}(\tau, \tau) .
\end{array}
$$

Therefore, we have

$$
\begin{gathered}
\left(\begin{array}{cc}
Q_{2} & A_{2}^{\prime} \\
A_{2} & -S_{2}
\end{array}\right)(t)-\left(\begin{array}{cc}
Q_{1} & A_{1}^{\prime} \\
A_{1} & -S_{1}
\end{array}\right)(t) \\
=\left(\begin{array}{cc}
\frac{1-\gamma}{2 \gamma} K^{2} \sigma_{r}^{2} & 0 \\
0 & 0
\end{array}\right) \geq \mathbf{0}_{2 \times 2},
\end{gathered}
$$

and $K_{2}(\tau) \geq K_{1}(\tau)$. Applying Proposition $9, k(t, \tau) \leq \widetilde{K}(t, \tau)$ for all $t \in[0, \tau]$. It is because $\sigma_{r}^{2} / 2 \geq 0$ and $-((1-$ $\gamma) / 2 \gamma) K^{2} \sigma_{r}^{2} \leq 0$ in $\operatorname{ODE}(72)$, and $k(\tau, \tau)=K(\tau, T) \geq 0$, which is proved in Lemma 10, that $k(t, \tau)$ exists for all $t \in$ $[0, \tau]$ by Theorem 4.1 in $[16]$.

Theorem 14. The function,

$$
\widetilde{v}(t, Y, r)=\frac{Y^{1-\gamma} \psi_{\mu^{*}(t, \tau)}\left(\psi_{z}(1-\gamma)-1\right)}{1-\gamma} e^{\widetilde{K}(t, \tau) r+\widetilde{M}(t, \tau)},
$$

is bounded for all $0 \leq t \leq \tau \leq T$ if $K(t, T)$ has no finite escape time on $[0, T]$.

Proof. Suppose that $K(t, T)$ has no finite escape time on $[0, T]$. It implies that $\widetilde{K}(t, \tau)$ are bounded for $0 \leq t \leq$ $\tau \leq T$, and so is $\widetilde{M}(t, \tau)$. Since the moment generating function of $\mu^{*}(t, \tau)$ and $z, \psi_{\mu^{*}(t, \tau)}(\cdot)$ and $\psi_{z}(\cdot)$, then $\widetilde{v}(t, Y, \beta)$ is bounded.

The above theorem shows the uniform integrability of $\left\{V\left(\tau, Y^{u^{*}}(\tau), \beta(\tau)\right\}_{\tau}\right.$. The calculation of

$$
\begin{gathered}
\mathrm{E}\left[\ln Y(\tau)+\int_{\tau}^{T} \frac{\|\Lambda(s)\|^{2}}{2} d s-\mathrm{E}[z] \int_{\tau}^{T} \mathrm{E}[\mu(s)] d s\right. \\
+\int_{\tau}^{T}\left(e^{-\int_{\tau}^{s} b(\eta) d \eta} r(\tau)+\int_{\tau}^{s} e^{-\int_{\eta}^{s} b(\theta) d \theta} a(\eta) d \eta\right) \\
\left.\times\left(1-\frac{\lambda_{r}(s)^{2}}{2}\right) d s \mid \mathscr{G}_{t}\right]
\end{gathered}
$$

will be shown as follows:

$$
\begin{gathered}
\mathrm{E}\left[\ln Y(\tau)+\int_{\tau}^{T} \frac{\|\Lambda(s)\|^{2}}{2} d s-\mathrm{E}[z] \int_{\tau}^{T} \mathrm{E}[\mu(s)] d s\right. \\
+\int_{\tau}^{T}\left(e^{-\int_{\tau}^{s} b(\eta) d \eta} r(\tau)+\int_{\tau}^{s} e^{-\int_{\eta}^{s} b(\theta) d \theta} a(\eta) d \eta\right) \\
\left.\times\left(1-\frac{\lambda_{r}(s)^{2}}{2}\right) d s \mid \mathscr{G}_{t}\right] \\
=\int_{\tau}^{T} \frac{\|\Lambda(s)\|^{2}}{2} d s-\mathrm{E}[z] \int_{\tau}^{T} \mathrm{E}[\mu(s)] d s \\
+\mathrm{E}\left[\ln Y^{u^{*}}(\tau)\right. \\
+\int_{\tau}^{T}\left(e^{-\int_{\tau}^{s} b(\eta) d \eta} r(\tau)+\int_{\tau}^{s} e^{-\int_{\eta}^{s} b(\theta) d \theta} a(\eta) d \eta\right) \\
\left.\times\left(1-\frac{\lambda_{r}(s)^{2}}{2}\right) d s \mid \mathscr{G}_{t}\right]
\end{gathered}
$$

$$
=\int_{\tau}^{T} \frac{\|\Lambda(s)\|^{2}}{2} d s-\mathrm{E}[z] \int_{\tau}^{T} \mathrm{E}[\mu(s)] d s
$$$$
+\mathrm{E}\left[\ln Y^{u^{*}}(\tau)\right.
$$

$$
\left.+\int_{\tau}^{T} \mathrm{E}\left[r(s) \mid \mathscr{G}_{\tau}\right]\left(1-\frac{\lambda_{r}(s)^{2}}{2}\right) d s \mid \mathscr{G}_{t}\right]
$$$$
=\int_{\tau}^{T} \frac{\|\Lambda(s)\|^{2}}{2} d s-\mathrm{E}[z] \int_{\tau}^{T} \mathrm{E}[\mu(s)] d s
$$$$
+\mathrm{E}\left[\ln Y^{u^{*}}(\tau) \mid \mathscr{G}_{t}\right]
$$$$
+\mathrm{E}\left[\int_{\tau}^{T} \mathrm{E}\left[r(s) \mid \mathscr{G}_{\tau}\right]\left(1-\frac{\lambda_{r}(s)^{2}}{2}\right) d s \mid \mathscr{G}_{t}\right]
$$$$
=\int_{\tau}^{T} \frac{\|\Lambda(s)\|^{2}}{2} d s-\mathrm{E}[z] \int_{\tau}^{T} \mathrm{E}[\mu(s)] d s
$$$$
+\ln Y(t)+\int_{t}^{\tau} \frac{\|\Lambda(s)\|^{2}}{2} d s-\mathrm{E}[z] \int_{t}^{\tau} \mathrm{E}[\mu(s)] d s
$$$$
+\int_{t}^{\tau} \mathrm{E}\left[r(s) \mid \mathscr{G}_{t}\right]\left(1-\frac{\lambda_{r}(s)^{2}}{2}\right) d s
$$$$
+\mathrm{E}\left[\int_{\tau}^{T} \mathrm{E}\left[r(s) \mid \mathscr{G}_{\tau}\right]\left(1-\frac{\lambda_{r}(s)^{2}}{2}\right) d s \mid \mathscr{G}_{t}\right]
$$$$
=\int_{t}^{T} \frac{\|\Lambda(s)\|^{2}}{2} d s-\mathrm{E}[z] \int_{t}^{T} \mathrm{E}[\mu(s)] d s+\ln Y(t)
$$$$
+\mathrm{E}\left[\mathrm{E}\left[\int_{t}^{T} r(s)\left(1-\frac{\lambda_{r}(s)^{2}}{2}\right) d s \mid \mathscr{G}_{\tau}\right] \mid \mathscr{G}_{t}\right]
$$$$
=\int_{t}^{T} \frac{\|\Lambda(s)\|^{2}}{2} d s-\mathrm{E}[z] \int_{t}^{T} \mathrm{E}[\mu(s)] d s
$$ 


$$
\begin{gathered}
+\ln Y(t)+\int_{t}^{T} \mathrm{E}\left[r(s) \mid \mathscr{G}_{t}\right]\left(1-\frac{\lambda_{r}(s)^{2}}{2}\right) d s \\
=\int_{t}^{T} \frac{\|\Lambda(s)\|^{2}}{2} d s-\mathrm{E}[z] \int_{t}^{T} \mathrm{E}[\mu(s)] d s+\ln Y(t) \\
+\int_{t}^{T}\left(e^{-\int_{t}^{s} b(\eta) d \eta} r(t)+\int_{t}^{s} e^{-\int_{\eta}^{s} b(\theta) d \theta} a(\eta) d \eta\right) \\
\quad \times\left(1-\frac{\lambda_{r}(s)^{2}}{2}\right) d s<+\infty .
\end{gathered}
$$

Hence, $\left\{J\left(\tau, Y^{u^{*}}(\tau), \beta(\tau)\right\}_{\tau}\right.$ is uniformly integrable.

\section{Conclusion}

We investigate the optimal investment of insurers with the extended CIR stochastic interest rate model by maximizing the expected utility on the insurer's terminal wealth. Using the HJB framework, the problem is converted into a PIDE for which we derive a closed-form explicit solution. A verification theorem is provided to offer conditions under which the solution of the PIDE is indeed the optimal value of the expected utility. Hence, the derived optimal control is indeed the optimal control for the original optimization problem. Future potential researches include incorporating longevity risk [17] into the ALM problem with CIR stochastic interest rate and extending the result to multivariate CIR or Wishart process as discussed in [18]. Techniques reported here are useful for establishing the verification theorem.

\section{Conflict of Interests}

The authors declare that there is no conflict of interests regarding the publication of this paper.

\section{Acknowledgments}

Mei Choi Chiu acknowledges the support of Research Grant Council of Hong Kong with ECS Project no. 809913. Hoi Ying Wong acknowledges the support of Research Grant Council of Hong Kong with GRF Project no. 403511.

\section{References}

[1] S. Thind, "Nordic insurers face up to low rates hedging challenge," 2013, http://www.risk.net/insurance-risk/feature/2263688/nordic-insurers-face-up-to-lowrates-hedging-challenge.

[2] J. C. Cox, J. E. Ingersoll, Jr., and S. A. Ross, "A theory of the term structure of interest rates," Econometrica, vol. 53, no. 2, pp. 385407, 1985.

[3] H. Y. Wong and M. C. Chiu, "Homotopy analysis method for boundary-value problem of turbo warrant pricing under stochastic volatility," Abstract and Applied Analysis, vol. 2013, Article ID 682524, 5 pages, 2013.

[4] G. Deelstra, M. Grasselli, and P.-F. Koehl, "Optimal investment strategies in a CIR framework," Journal of Applied Probability, vol. 37, no. 4, pp. 936-946, 2000.
[5] G. Deelstra, M. Grasselli, and P.-F. Koehl, "Optimal investment strategies in the presence of a minimum guarantee," Insurance: Mathematics \& Economics, vol. 33, no. 1, pp. 189-207, 2003.

[6] R. Ferland and F. Watier, "Mean-variance efficiency with extended CIR interest rates," Applied Stochastic Models in Business and Industry, vol. 26, no. 1, pp. 71-84, 2010.

[7] H. Chang and X.-M. Rong, "An investment and consumption problem with CIR interest rate and stochastic volatility," Abstract and Applied Analysis, vol. 2013, Article ID 219397, 12 pages, 2013.

[8] H. Yang and L. Zhang, "Optimal investment for insurer with jump-diffusion risk process," Insurance: Mathematics \& Economics, vol. 37, no. 3, pp. 615-634, 2005.

[9] M. C. Chiu and H. Y. Wong, "Mean-variance asset-liability management: cointegrated assets and insurance liability," European Journal of Operational Research, vol. 223, no. 3, pp. 785793, 2012.

[10] M. C. Chiu and H. Y. Wong, "Optimal investment for an insurer with cointegrated assets: CRRA utility," Insurance: Mathematics \& Economics, vol. 52, no. 1, pp. 52-64, 2013.

[11] C. Hipp and M. Plum, "Optimal investment for insurers," Insurance: Mathematics \& Economics, vol. 27, no. 2, pp. 215-228, 2000.

[12] W. H. Fleming and H. M. Soner, Controlled Markov Processes and Viscosity Solutions, vol. 25, Springer, New York, NY, USA, 1993.

[13] H. Abou-Kandil, G. Freiling, V. Ionescu, and G. Jank, Matrix Riccati Equations in Control and Systems Theory, Birkhäuser, Basel, Switzerland, 2003.

[14] G. Freiling and G. Jank, "Generalized Riccati difference and differential equation," Linear Algebra and Its Applications, vol. 241-243, pp. 291-303, 1996.

[15] J. Gallier, "The Schur complement and symmetric positive semidefinite (and definite) matrices," 2010, http://www .cis.upenn.edu/ jean/schur-comp.pdf.

[16] G. Freiling, G. Jank, and A. Sarychev, "Lyapunov-type functions and invariant sets for Riccati matrix differential equations," in Proceedings of ECC, B. van Dooren, Ed., Brussels, Belgium, 1997.

[17] T. W. Wong, M. C. Chiu, and H. Y. Wong, "Time-consistent mean-variance hedging of longevity risk: effect of cointegration," Insurance: Mathematics and Economics, vol. 56, pp. 56-67, 2014.

[18] M. C. Chiu and H. Y. Wong, "Mean-variance portfolio selection with correlation risk," Journal of Computational and Applied Mathematics, vol. 263, pp. 432-444, 2014. 


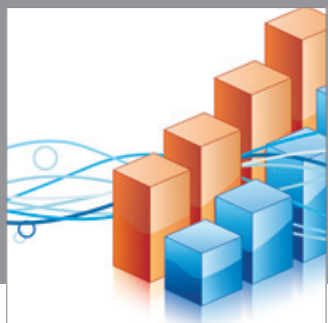

Advances in

Operations Research

mansans

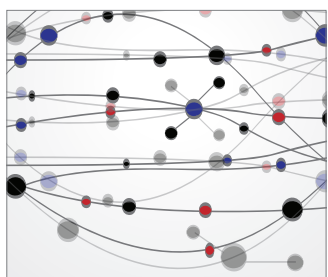

The Scientific World Journal
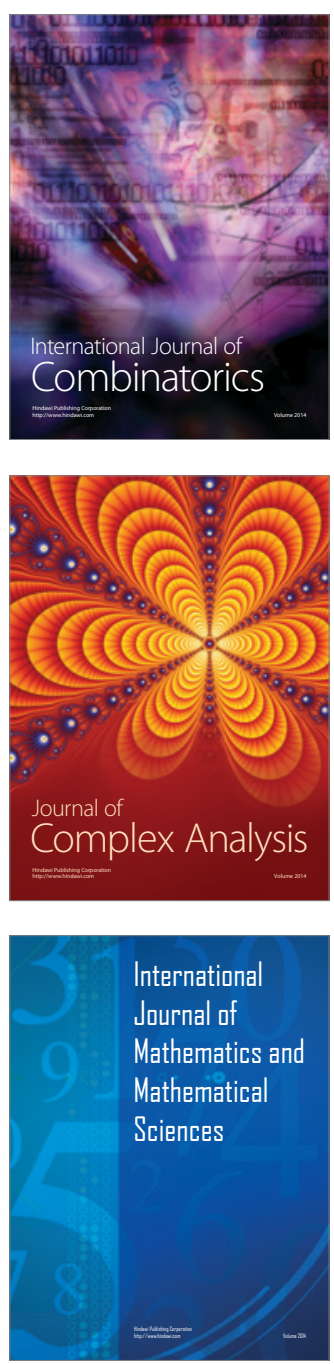
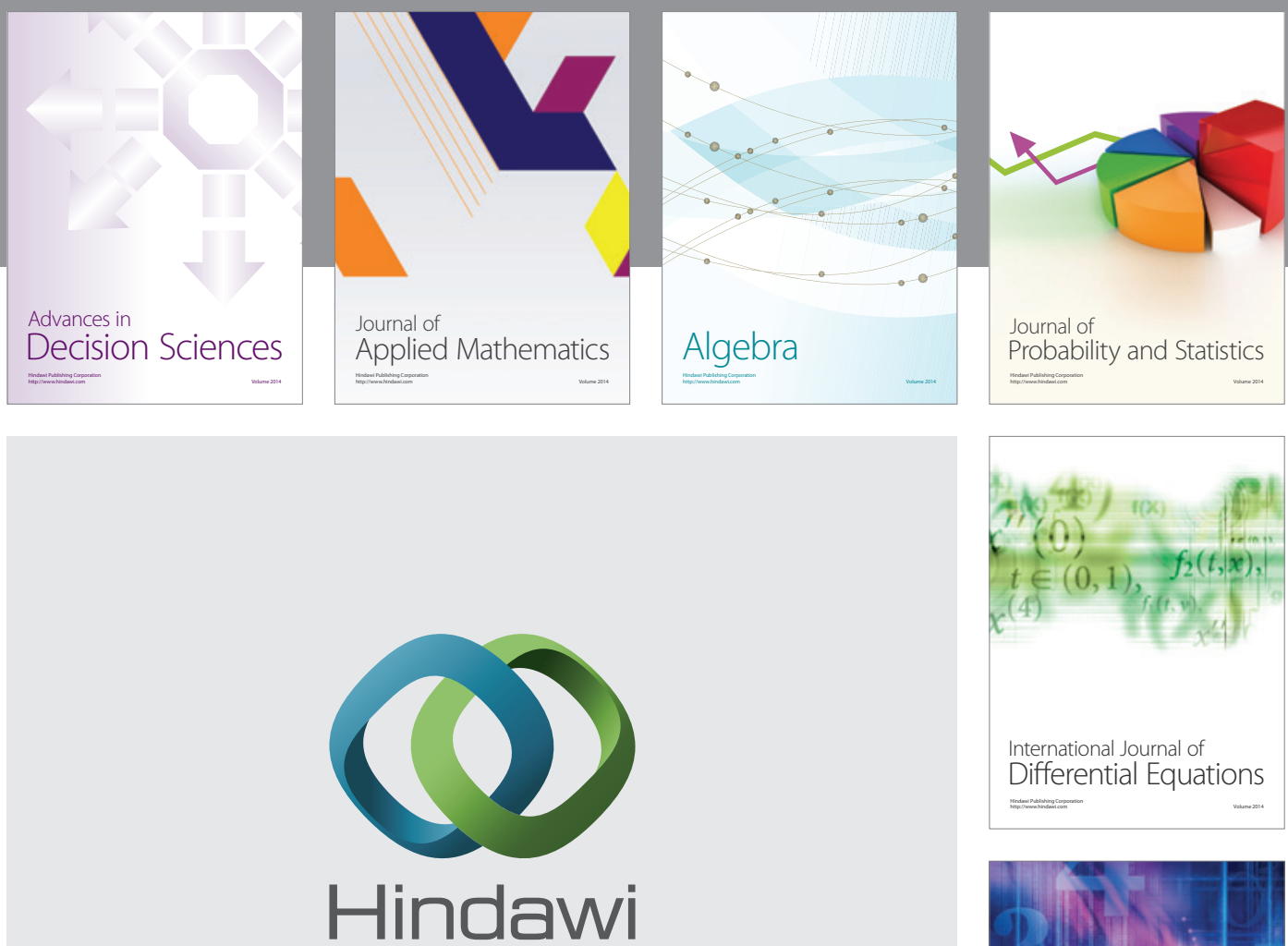

Submit your manuscripts at http://www.hindawi.com
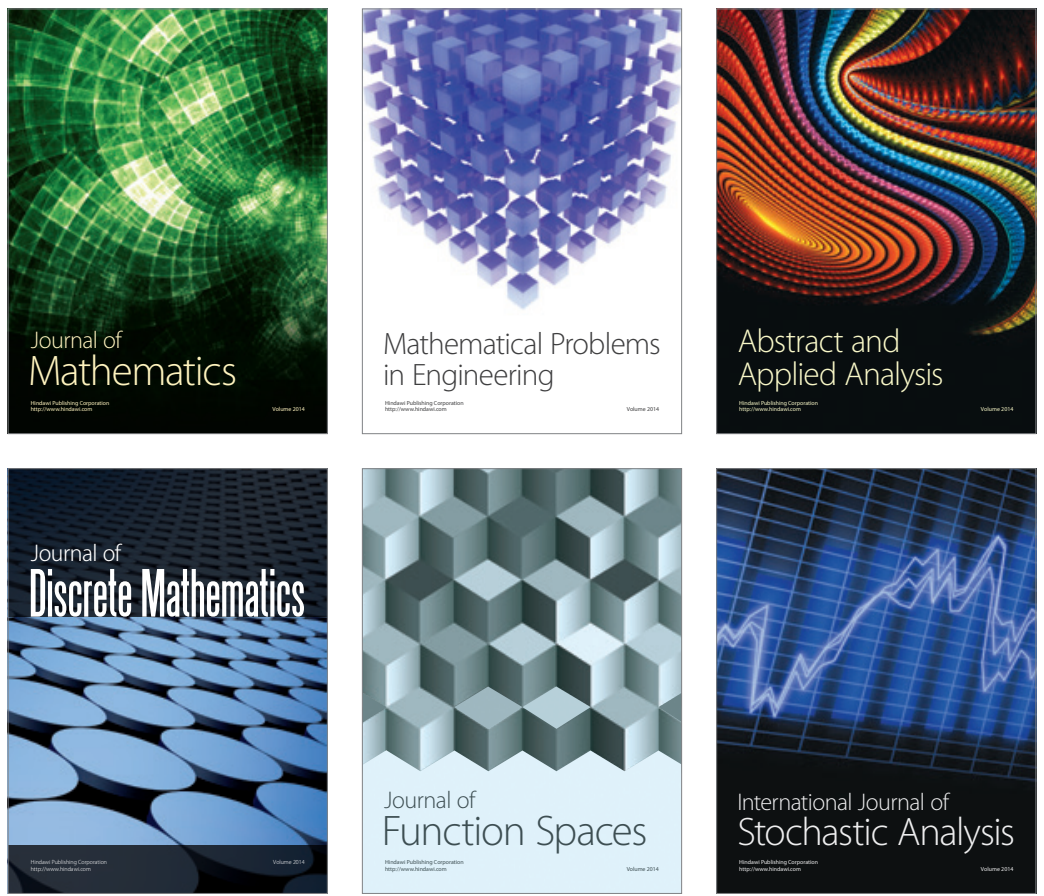

Journal of

Function Spaces



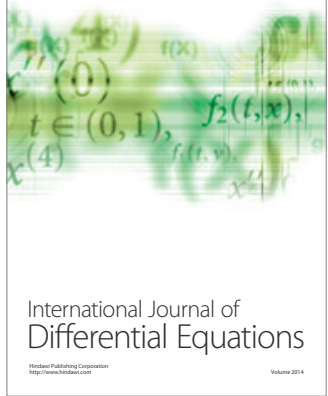
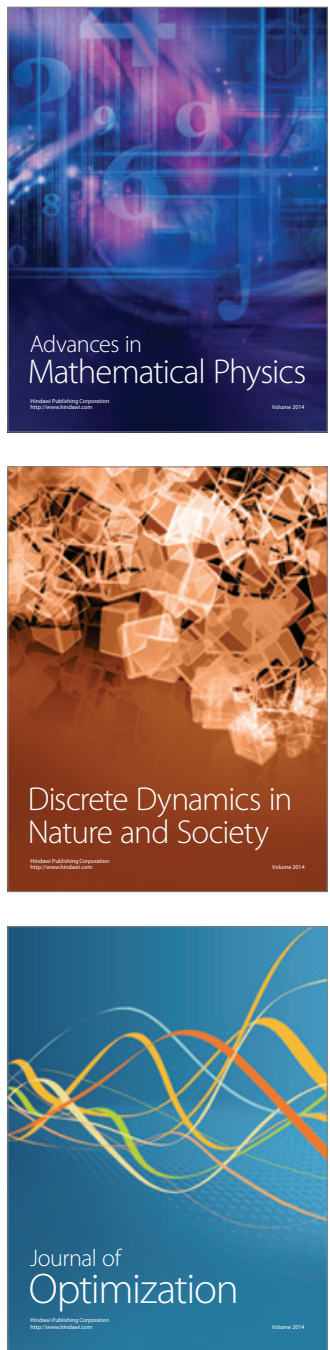